\title{
Las ideas y las prácticas. Agenda de gobierno y régimen de políticas universitarias en México
}

\author{
Adrián Acosta Silva \\ Sociólogo. Coordinador académico de la Unidad de Política Educativa del Centro de \\ Estudios Estratégicos para el Desarrollo de la Universidad de Guadalajara.
}

\begin{abstract}
Resumen
Este texto es un esfuerzo por identificar la agenda, los cambios y las tensiones observadas en la primera etapa del gobierno federal encabezado por Andrés Manuel López Obrador en el Campo Universitario Mexicano. El análisis descansa en la perspectiva de "Régimen de Políticas", que focaliza la atención en las ideas, los arreglos institucionales y los actores principales en el diseño e implementación de las políticas públicas. El argumento central a discusión es que el nuevo gobierno no se ha orientado, hasta el momento, en la construcción de un nuevo régimen de políticas, mostrando una clara tendencia hacia la continuidad de políticas anteriores, incluso un nuevo contexto político, de austeridad financiera y redistribución presupuestal de los programas y recursos públicos en la educación superior.
\end{abstract}

Palabras clave: México, Universidades públicas, Agenda de gobierno, Régimen de políticas, Gobernanza. 


\title{
As ideias e as práticas. Agenda de governo e regime de políticas universitárias no México
}

\section{Sumario}

O texto é um esforço por identificar a agenda, as mudanças e as tensões observadas na primeira etapa do governo federal liderado por Andrés Manuel López Obrador no Campo Universitário Mexicano. A análise parte da perspectiva do "Regime das Políticas", que tem o foco na atenção das ideias, nos arreglos institucionais e nos atores principais do design e da implementação das políticas públicas. O argumento central da discussão é que o novo governo não se tem orientado até o momento na construção de um novo regime de políticas, mostrando uma tendência clara com a continuidade das políticas anteriores, e também um novo contexto político, de austeridade financeira e redistribuição orçamental dos programas e recursos públicos na Educação Superior.

Palavras-chave: México, Universidades Públicas, Agenda de Governo, Regime de Políticas, Governança.

\section{Ideas and practices: Political Agenda and University Policy Regime in Mexico}

\begin{abstract}
This is an effort to identify the agenda, changes and tensions concerning the Mexican university field that have been observed during the first stage of Andres Manuel López Obrador's federal government administration. The analysis lies in the policy regime perspective, which focuses on the ideas, the institutional arrangements and the main actors of the public policies design and implementation. The main argument under discussion is that the new government has not yet centred on the construction of a new policy regime, a fact that shows an explicit tendency towards the previous policies continuity, but at the same time towards a new political context of financial austerity and a budgetary redistribution of higher education public resources and programs.
\end{abstract}

Keywords: Mexico, Public universities, Political agenda, Policy regime, Governance. 


\section{Introducción}

El 1 de diciembre de 2018 tomó posesión como Presidente de la República Andrés Manuel López Obrador (AMLO). Luego de una elección ganada con casi 30 millones de votos (53\% de la votación total), y donde su partido político logró obtener la mayoría relativa de los escaños tanto en la cámara de diputados (38\%) como en la de senadores (43\%), la elección significaba no sólo el triunfo contundente de una organización política relativamente nueva (el Movimiento de Regeneración Nacional, MORENA), sino el fin del ciclo de los "gobiernos divididos" (un congreso no dominado por el partido del presidente) que habían caracterizado la alternancia política en México desde el 2000, y el regreso al "gobierno unificado" (un congreso dominado mayoritariamente por el partido del presidente) que existía hasta antes de 1997.

Más allá de la combinación de motivaciones y factores causales que explican el triunfo de AMLO, lo relevante es que el origen de la legitimidad democrática del nuevo gobierno quedó fuera de toda duda. Eso permitió la llegada al poder de un nuevo oficialismo en el gobierno federal que emprendería una "transformación profunda" de México, un "renacimiento" capaz de resolver los problemas de desigualdad social y pobreza que desde su perspectiva política caracterizan la historia reciente del país. Ese "renacimiento" se concentró en el impulso a una idea que el oficialismo denomina como la "Cuarta Transformación Nacional", un proyecto que identifica la corrupción política y el neoliberalismo económico como las causas fundamentales de los grandes problemas nacionales, causalidades que pueden ser resueltas mediante políticas de austeridad, redistribución de los recursos públicos, "honestidad republicana" y la construcción de una "verdadera democracia" (AMLO, 2017).

En ese contexto, la retórica y la acción gubernamental se han concentrado en el cambio del régimen político y de políticas a través del ejercicio de los poderes federales en los diversos campos de la acción pública. La lógica política del lopezobradorismo -como suele denominarse a la heterogénea coalición de creencias, intereses e ideas que imprimen cohesión y fuerza política al nuevo oficialismo-, se explica mediante el ejercicio de un fuerte liderazgo personalista/presidencial en la construcción de acuerdos políticos, la formación de arreglos institucionales y la instrumentación de políticas gubernamentales que fortalecen iniciativas de cambio en los diversos campos de la vida pública.

El sector universitario forma parte de esos ámbitos de la acción pública. ¿Cuál es la agenda del nuevo gobierno frente a las universidades? ¿Qué cambios se han instrumentado o se perfilan en ese sector? ¿Qué tipo de tensiones pueden advertirse en las relaciones entre el oficialismo y las universidades? La perspectiva de análisis que orienta la exploración de estas cuestiones es la del "Régimen de Políticas", un enfoque que concentra la atención en tres componentes básicos: las ideas, los arreglos institucionales, y los actores de las políticas. A menos de dos años de iniciado el ejercicio gubernamental es posible formular algunas ideas e hipótesis sobre la gestión del nuevo gobierno federal mexicano en el campo de las políticas universitarias. Ese es el propósito central de este artículo. 
Para ello, el texto se organiza en tres secciones. En la primera se analiza, en contexto, las políticas educativas universitarias del gobierno de AMLO, revisando los antecedentes del "viejo régimen" dominante durante tres décadas (1989-2018). En la segunda, se describe cuál es el diagnóstico y la agenda gubernamental para la educación superior del nuevo gobierno. Finalmente, se ofrecen algunas consideraciones a manera de conclusiones.

\section{El contexto de las políticas}

Las políticas y sus prácticas nunca surgen en un vacío histórico o institucional. Siempre existen ideas, arreglos y actores interesados en la promoción y búsqueda de resultados esperados en los campos específicos de la acción pública que generan a lo largo del tiempo hábitos, rutinas y "sistemas de creencias" que determinan, influyen o condicionan los comportamientos institucionales. La acción pública puede ser conceptualizada como resultado de la implementación de un "régimen de políticas" configurado por la relación entre determinadas ideas, arreglos institucionales y actores estratégicos involucrados en el proceso de las políticas (May, 2018). La premisa básica del enfoque es que todo régimen de políticas produce experiencias, acumula legados, legitima prácticas, establece rutinas de negociación, privilegia actores relevantes y agendas prioritarias que configuran un orden institucional más o menos estable a lo largo de un periodo determinado (Wilson, 2000).

La educación superior mexicana contemporánea puede ser analizada desde esta perspectiva analítica. Luego de tres administraciones gubernamentales federales marcadas por la alternancia $(2000,2006,2012)$, la educación universitaria se ha vuelto territorio marcado por las huellas de las políticas públicas instrumentadas a lo largo de la primera parte del siglo XXI. Durante tres sexenios dominados por oficialismos distintos (20002012, PAN, y 2012-2018, PRI), ese territorio fue conquistado por la irrupción de una nueva fuerza política (MORENA), que en julio de 2018 triunfó abrumadoramente en las elecciones presidenciales, proponiendo una ruptura radical con el pasado "neoliberal" -o "neoporfirista", como suele denominarlo el nuevo oficialismo-, colocando en el centro de su retórica y acción como gobierno federal la propuesta de una "transformación histórica" del país, denominada "Cuarta Transformación Nacional" (4T), que incluye un nuevo modelo de políticas educativas ${ }^{1}$.

El gobierno del presidente López Obrador, electo para el periodo 20182024, es una expresión política compleja que combina rasgos democráticos, populistas y autocráticos. A lo largo de las tres últimas décadas, su liderazgo es producto del tránsito por partidos políticos como el PRI en su natal estado de Tabasco, luego, a nivel nacional, por el PRD (partido del cual AMLO fue su dirigente nacional, y posteriormente candidato electo Jefe de Gobierno del Distrito Federal en el periodo 2000-2006, así como candidato presidencial en las elecciones federales de 2006 y 2012), al cual renunció en 2013 para fundar el actual partido, MORENA, en 2014.

El morenismo es un movimiento hecho a la medida por su líder, una hechura que combina prácticas clientelares y populistas del viejo PRI, con la retórica izquierdista contra la pobreza, la desigualdad y la antidemocracia 
mexicana, al que se añade también valores como la fe, la honestidad, la solidaridad y creencias relacionadas con el amor al prójimo, la felicidad del pueblo y la fraternidad nacional. Esos son algunos puntos que unen las poliformes costuras ideológicas del nuevo oficialismo. Durante su campaña electoral, el ya candidato presidencial planteó con claridad el núcleo de sus ideas y propósitos:

La crisis de México no podrá enfrentarse sin cortar de tajo con la corrupción y la impunidad, lo cual implica cambiar el actual régimen y establecer un orden político nuevo, democrático, promotor de la legalidad, humanista y con el distintivo de la honestidad. Hoy existe una república simulada, no un gobierno del pueblo y para el pueblo. El Estado ha sido convertido en un mero comité al servicio de una minoría rapaz y, como decía Tolstói, un Estado que no procura la justicia no es más que una banda de malhechores (AMLO, 2017:13).

La narrativa rupturista constituye el centro simbólico del lopezobradorismo. Desde el inicio de su gestión, el nuevo gobierno nacional ha impulsado algunos cambios normativos y organizacionales en la educación superior universitaria, pero también ha mantenido las rutinas e inercias asociadas a las políticas del pasado reciente. Esa combinación de intenciones de cambio e inercias institucionales son el efecto de una agenda política que obedece a un "sistema de creencias" más que a un "sistema de evidencias", lo que dificulta identificar el ideario oficialista en educación universitaria, ¿cuál es su origen y cómo se articula regionalmente con otras experiencias latinoamericanas contemporáneas?

\section{El "viejo régimen" de políticas (1989-2018)}

Las agendas universitarias del largo ciclo anterior (1989-2018) estuvieron marcadas por dos ejes compartidos por las diversas administraciones sexenales: la evaluación de la calidad y el financiamiento público condicionado, diferencial y competitivo. Varios programas federales se instrumentaron a lo largo del tiempo, influyendo en el comportamiento institucional de las nueve universidades públicas federales y 34 estatales que constituyen el núcleo organizativo, académico y científico del Sistema Nacional de Educación Superior en México.

Ese ciclo generó una estructura de políticas federales basadas en el uso intensivo de incentivos y restricciones que explica las lógicas institucionales del funcionamiento de las universidades públicas. Internacionalización, acreditación y aseguramiento de la calidad, productividad académica, rendición de cuentas, evaluación del desempeño institucional, reforma del gobierno universitario, fueron los temas centrales de las agendas universitarias. La búsqueda y construcción de indicadores de calidad se convirtió en el centro de acción de los gobiernos universitarios, indicadores que permitieran acceder a mayores recursos públicos a través de las bolsas de financiamiento federal extraordinario diseñadas para estimular cambios en los comportamientos de las universidades públicas (Acosta, 2014).

Se construyó así una suerte de "épica de los indicadores" como narrativa dominante de las políticas universitarias (Acosta, 2019a). Como relato 
y práctica institucional, esa épica sustituyó a la "épica autonómica" que dominó el comportamiento universitario desde la reforma de Córdoba de 1918 y la primera autonomía de la UNAM en 1929, hasta finales de los años ochenta, para luego transitar por la "épica de la modernización” surgida desde los años noventa impulsada por los gobiernos de Carlos Calinas de Gortari (1988-1994) y de Ernesto Zedillo (1994-2000), los últimos gobiernos del PRI durante el siglo XX. En la era de las alternancias (2000-2018), el énfasis en la acreditación y aseguramiento de la calidad promovida tanto por los gobiernos panistas (Vicente Fox, 2000-2006; Felipe Calderón, 20122018), como por el retorno del priismo (Enrique Peña Nieto, 2012-2018), conservó las políticas de financiamiento y evaluación, traducidas como “épicas de la calidad". Estas épicas fueron traducidas como regímenes de políticas, dirigidas a construir representaciones sobre el prestigio, la reputación y la legitimidad institucional de las universidades públicas frente a los diversos gobiernos nacionales y, de manera secundaria, frente a sus propias sociedades locales y comunidades universitarias. En ese contexto, una suerte de legitimidad bifronte, siempre en tensión, fue el resultado de las épicas y agendas de políticas experimentadas a lo largo de los últimos treinta años en México.

El origen de esas agendas del pasado reciente tuvo un claro componente internacional, derivado de recomendaciones de organismos internacionales (Banco Mundial, UNESCO, OECD), la experiencia del "Estado evaluador" europeo, y la americanización de la educación superior, es decir, la experiencia de las grandes universidades de investigación de la Costa Este de los Estado Unidos de Norteamérica como modelos del cambio universitario mexicano. Pero también existió un componente nacional, que tuvo que ver con la crisis del financiamiento público a las universidades de los años ochenta ("la década perdida"), los conflictos políticos de los años noventa (procesos de reforma institucional en varias universidades públicas tanto federales como estatales), y los cambios en los patrones o estilos de conducción política tanto de las políticas públicas como de los gobiernos universitarios.

El régimen de políticas surgido de este ciclo largo combinó la interacción de un par de ideas (calidad y evaluación), arreglos institucionales (normatividad, financiamiento, gobernanza) y actores interesados (Gobierno federal, Cámara de Diputados, Gobernadores, ANUIEs, rectores universitarios, organismos acreditadores), instrumentando a lo largo del tiempo diversos programas federales en la gestión de las universidades públicas (Acosta, 2010). El nuevo oficialismo ha definió ese ciclo de políticas como "neoliberal", causal fundamental de los problemas de cobertura, eficiencia y eficacia social de las universidades. En su lugar, propone un cambio sustancial en el perfil, contenido y orientación de las políticas hacia la educación superior, es decir, la construcción de un régimen de políticas basado en ideas, arreglos y actores distintos a los dominantes durante casi tres décadas. Esa tríada se perfila como una relación entre las ideas de la "obligatoriedad" y "gratuidad" de la educación superior, arreglos institucionales basados en una redistribución de las prioridades del financiamiento público concentradas en dos programas estratégicos (Jóvenes escribiendo el futuro, y Universidades para el Bienestar), y el impulso a una reforma a la legislación federal (la Ley General de Educación Superior), donde los actores centrales son el Ejecu- 
tivo federal, el Congreso, y las autoridades y directivos de las instituciones públicas de educación superior.

Pero tanto el viejo régimen como el proclamado nuevo régimen de políticas universitarias no se comprenden en sí mismos, sino con referencia al tipo de relaciones que guardan con el Estado. Resulta interesante mirar cómo, en el caso de las universidades públicas, tanto las narrativas modernizadoras de los pasados oficialismos basadas en ideas de la calidad y evaluación, como la narrativa del nuevo oficialismo, a su vez basada en la idea de gratuidad y obligatoriedad de la educación superior, comparten el mismo principio de políticas públicas: la desconfianza en la autonomía de las universidades. El viejo régimen introdujo mecanismos de evaluación, acreditación y aseguramiento de la calidad por el escepticismo gubernamental en el desempeño y los resultados de las universidades tradicionales. El nuevo régimen se orienta a la creación de un nuevo sistema federal de universidades públicas porque no confía en que la autonomía universitaria pueda resolver los problemas de equidad y cobertura en el acceso y egreso de la educación superior. El hilo político conductor de ambos regímenes tiene que ver con el recelo de los oficialismos correspondientes respecto del ejercicio de la autonomía universitaria frente a entornos de estatalidades complejas, cambiantes y contradictorias (Acosta, 2020). 


\section{El diagnóstico y las propuestas}

Para el oficialismo contemporáneo, las políticas "neoliberales" son la causa fundamental de los problemas de inequidad, ineficiencia y bajo impacto de la educación superior en el empleo profesional y la movilidad social de los egresados. Esas políticas estructuraron un mismo modelo de intervenciones públicas construido a lo larsgo de los últimos años por diversos gobiernos nacionales que llevaron a la "pérdida de la gratuidad de la educación superior", "se sometió a las universidades públicas a un acoso presupuestal sin precedentes" y "se excluyó a miles de jóvenes en las universidades". En los hechos, "ello no sólo privó al país de un número incalculable de graduados, sino que agravó el auge de la delincuencia y las conductas antisociales..." (PND, 2019: p.50).

Bajo este diagnóstico, la agenda del nuevo oficialismo definió dos instrumentos centrales para transformar la educación superior universitaria y no universitaria en México: el programa Jóvenes Escribiendo el Futuro (JEF), dirigido a estudiantes universitarios mayores de 18 y menores de 29 años de edad inscritos en instituciones públicas, y el programa Universidades para el Bienestar Benito Juárez García (UBBJG), iniciado en marzo de 2019, y que contempla inicialmente la apertura de 100 escuelas ubicadas en 31 de las 32 entidades (Estados) del país, para alcanzar a una población de 32 mil estudiantes para el año 2024, todos de tiempo completo, ubicados en zonas de alta marginalidad, que ingresan sin examen de admisión y reciben a lo largo de su formación una beca de 2,400 pesos mensuales (equivalentes a 100 dólares americanos).

Ambos programas son de carácter prioritario para el gobierno federal, y dependen fundamentalmente tanto de la Secretaría del Bienestar Social como de la Secretaría de Educación Pública, donde tradicionalmente se ubican los programas públicos de educación superior. Ese giro no obedece solamente a una reorganización interburocrática, sino que muestra el interés presidencial de colocar sus proyectos como parte de la política social dirigida a los sectores vulnerables de la población joven, más que una política educativa dirigida a los jóvenes en general. En ambos programas, el mecanismo de apoyo es directo a los individuos, para que reciban las becas federales "sin intermediarios", con el propósito de evitar prácticas de corrupción o ineficacias burocráticas indeseables (PSE, 2020: p.4).

Las ideas que gobiernan esas propuestas y proyectos son vagas tanto en su origen como en su consistencia explicativa. Es posible advertir una lógica populista ("primero los pobres") que coexiste con una lógica neoliberal (transferencias monetarias directas a los individuos más que a las instituciones). Esa mixtura tiene que ver con los problemas de la equidad en el acceso, el egreso y la universalización de la educación superior que afectan la cobertura e inclusión de la educación universitaria. Como en administraciones anteriores, las prioridades y mecanismos del nuevo gobierno, para instrumentar los principios de obligatoriedad y gratuidad, están fuertemente relacionadas con cuatro factores clave que explican el impulso a esas ideas y su traducción política como parte de la agenda 2018-2024: 1) Los déficits en la tasa bruta de cobertura de la educación superior (que en México alcanza sólo 38.6\% de los jóvenes en edad correspondiente, 18-22 
años; 2) los problemas de acceso a la educación superior (únicamente ingresan cada año entre 50 y $60 \%$ de los egresados del nivel medio superior a las universidades públicas); 3) la tasa de eficiencia terminal en los estudios universitarios es en promedio de 70\%; y, 4) la sobrerepresentación de los jóvenes de origen social "alto" y "medio" (definido por los deciles de ingreso familiar y la escolaridad de los padres) y la subrepresentación de los jóvenes de origen social "bajo" en las universidades públicas (Acosta y Planas, 2014).

En un sistema configurado por 3760 instituciones de educación superior (públicas y privadas, universitarias y no universitarias), en las que estudian poco más de 4.5 millones de estudiantes y laboran 414 mil profesores, las definiciones políticas y de políticas universitarias son clave para comprender cuál es la lógica institucional que propone el nuevo oficialismo. Siendo un sector heterogéneo, diverso y extraordinariamente complejo, la experiencia acumulada de las políticas anteriores muestra un sector sobreregulado por las políticas gubernamentales federales (el sector público dominado por las universidades federales y estatales, y los institutos tecnológicos federales), y un sector claramente subregulado (las universidades privadas).

En esas circunstancias, las becas para estudiantes y la creación de un nuevo sector de universidades públicas constituyen las apuestas centrales del gobierno lopezobradorista para la construcción de un nuevo régimen de políticas ${ }^{2}$. No obstante, en el viejo régimen se instrumentaron propuestas similares. En el pasado reciente ya existía un sistema federalizado de becas (Programa Nacional de Becas, PRONABES, fundado durante el periodo del foxismo -2000 a 2006-, y que sobrevivió con algunos ajustes hasta 2018), y la creación de un conjunto de universidades públicas e institutos tecnológicos (Universidades Interculturales, Universidades Públicas Estatales de Apoyo Solidario, Tecnológico Nacional de México) que fueron creados, apoyados o expandidos durante los sexenios 2006-2012 y 20122018. Para el resto de las instituciones y establecimientos públicos y para el sector privado, las reglas (las políticas) caminan por el mismo rumbo de los gobiernos pasados: diferenciación de programas de financiamiento ordinario y extraordinario para el sector público, y subregulación para las IES del sector privado como una forma de estimular su expansión nacional.

¿Cuál es el origen de esas ideas? El "sistema de creencias" del oficialismo hacia el sector parte de una clara desconfianza o un franco escepticismo respecto de la autonomía de las universidades públicas y de sus capacidades de transformación, una actitud que, paradójicamente, lo une a las políticas modernizadoras y evaluadoras de los gobiernos que le antecedieron. Bajo la retórica de la Cuarta Transformación, el regreso del hiperpresidencialismo mexicano significa un proceso de centralización de decisiones y recursos en el ejecutivo federal, que se traduce en un virtual estancamiento del financiamiento a las universidades públicas tradicionales, pero en un apoyo financiero a las Universidades del Bienestar durante 2019 y 2020 . En el viejo régimen, el déficit presupuestario federal hacia las universidades públicas se constituyó como una seña de identidad de las políticas modernizadoras y de las políticas de la calidad, lo que constituyó una de las razones por las cuales las universidades demandaban "certeza presupuestal" 


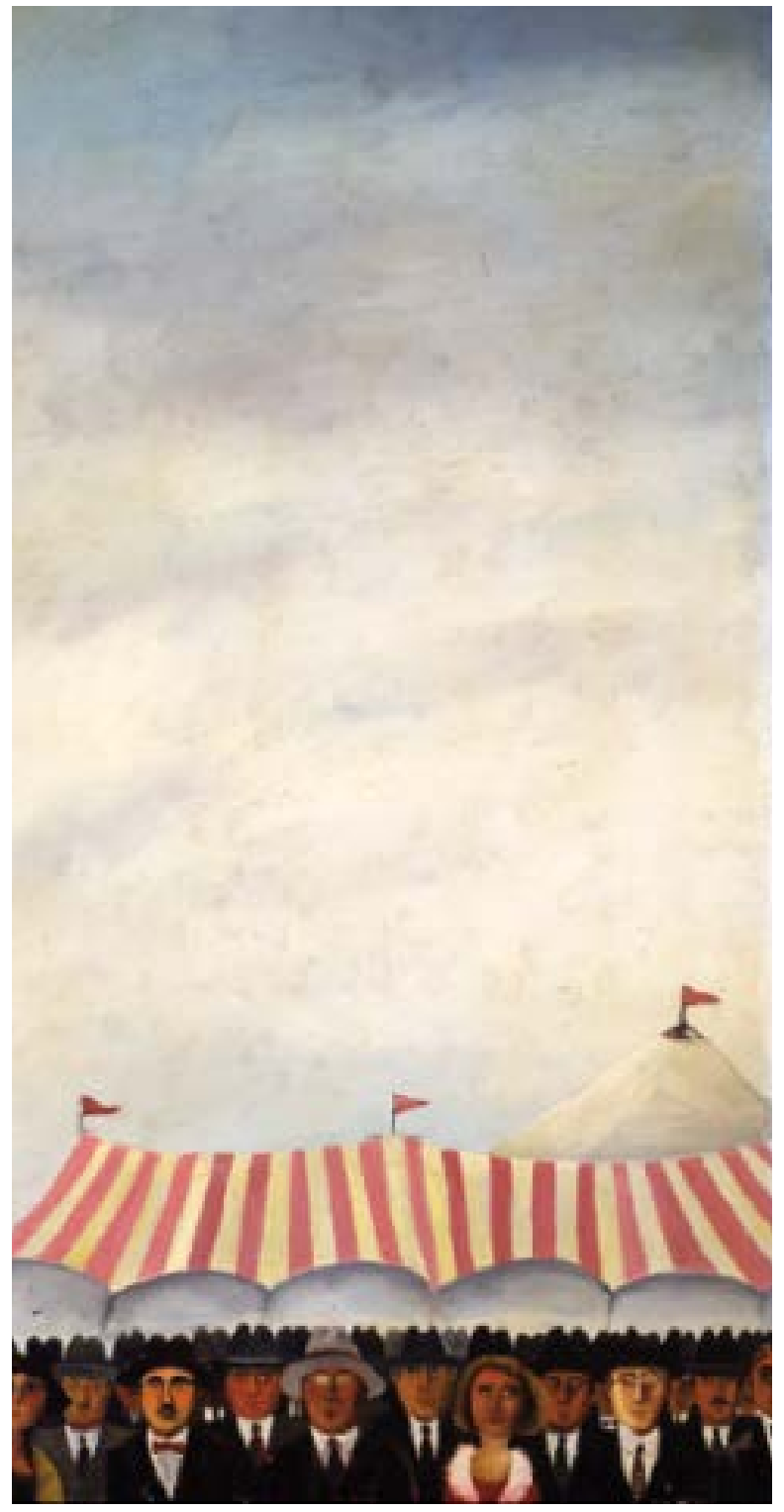

La feria internacional del sombrero (detalle). Óleo sobre tela, $90 \times 90 \mathrm{~cm} .1977$.

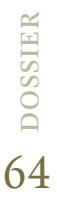

y "presupuestos multianuales" (ANUIES, 2018).

Otra creencia importante del oficialismo es que las universidades públicas no aportan resultados significativos a la disminución de los índices de desigualdad, inequidad y pobreza de la mayor parte de la población, debido, en parte, a las políticas de calidad centradas en la aplicación de exámenes de selección de estudiantes, que impiden el acceso a la educación universitaria de jóvenes con bajos niveles socioeconómicos. A pesar de los matices y las evidencias en contra, esa creencia parece explicar el escepticismo oficialista hacia el sector público universitario tradicional ${ }^{3}$.

En síntesis, las creencias que gobiernan el imaginario y las políticas del nuevo gobierno se presentan como parte de una transformación profunda de la educación superior, coherente con el proyecto de la $4 \mathrm{~T}$ que constituye el principio político distintivo del oficialismo. Estas creencias permiten identificar los conceptos de "excelencia", "obligatoriedad" y "gratuidad" como parte del ideario lopezobradorista en educación superior. Los arreglos institucionales suponen una pérdida relativa de las autonomías universitarias, privilegiando la heteronomía de las UBBJG. Los actores fundamentales son el gobierno federal, el congreso y los directivos de las instituciones públicas de educación superior. Estos elementos configuran la promesa de un nuevo régimen de políticas.

4 Universidades $\mid$ núm. 86, octubre-diciembre 2020| UDUAL $\mid$ DOI:https://doi.org/10.36888/udual.universidades.2020.86.405 Adrián Acosta Silva $\mid$ Las ideas y las prácticas. Agenda de gobierno y régimen de políticas universitarias en México 
La gran paradoja es que a pesar de la retórica del cambio de las políticas, lo que se observa es una fuerte continuidad de los instrumentos de las políticas, instrumentos que descansan en una clara lógica de incentivos y restricciones al comportamiento de las universidades. El uso del mismo instrumental básico de las políticas anteriores supone compartir ideas, orientaciones y estilos de conducción de las políticas propias. Es una paradoja clásica: los llamados al cambio se traducen en instrumentos orientados a la consolidación de comportamientos conservadores (Capano, 2011).

\section{Los instrumentos: intenciones y acciones}

Se pueden identificar 10 programas específicos distintos a los programas JEF y UBBJG, que organizan la acción federal hacia la educación superior, concentrados en la Dirección General de Educación Superior Universitaria (DGESU) de la SEP. La mayor parte de ellos ya existían desde hace años: "Programa de Apoyo al Desarrollo de la Educación Superior" (PADES), "Programa para el Desarrollo Profesional Docente para el tipo superior" (PRODEP), "Programa de Carrera Docente en Universidades Públicas Estatales" (U040), o el de "Subsidios Federales para Organismos Descentralizados Estatales". La única novedad es el "Programa de Fortalecimiento a la Excelencia Educativa" (PROFEXCE), que se basa en cuatro "ejes de acción": "compromiso social de las IES", "cobertura con equidad", "gobernanza del sistema de educación superior", y "financiamiento de la educación superior". (DGESU, 2020)

La meta de cobertura (TBC) en educación superior planteada en el "Programa Sectorial de Educación 2020-2024" (PSE) es llegar a 50.8\% para 2024, diez puntos porcentuales a la existente en 2019 (PSE, 2020). Evidentemente, esa meta y el propio PSE fueron elaborados antes de la crisis sanitaria y económica provocada por la pandemia de la COVID-19. Pero aún sin un escenario de crisis, el tamaño de los recursos necesarios y los cambios en los comportamientos institucionales para alcanzar esa tasa en tan sólo cinco años, mediante la dotación de becas a 300 mil estudiantes y ampliando las capacidades y ofertas públicas y privadas de ese nivel, resulta difícil de alcanzar. Según los cálculos realizados por la propia Subsecretaría de Educación Superior de la SEP, alcanzar el porcentaje de cobertura planeado como meta sexenal (50.8\%) significa pasar de una matrícula actual (ciclo escolar 2019-2020) de 4.5 a 6.13 millones en 2024, es decir, implicaría un crecimiento promedio anual de 298 mil nuevos estudiantes cada año (Mendoza Rojas, 2019a) ${ }^{4}$. Sin embargo, en el PSE esa meta de cobertura disminuye al 50\%, que equivale a 5.5 millones de estudiantes para 2024 (PSE, 2020:p.159).

En el PSE las políticas universitarias son contempladas en el marco más amplio de las políticas de educación superior. Mediante la definición de seis prioridades, los objetivos, metas, parámetros y resultados a alcanzar se definen mediante el enunciado de cientos de indicadores de gestión que constituyen la base de las métricas con las cuales el gobierno federal evaluará regularmente los avances del Programa a lo largo de los próximos cuatro años. Las prioridades son enunciadas de la siguiente manera: 1) "Educación para todas y todos, sin dejar a nadie atrás; 2) Educación de 
excelencia para aprendizajes significativos; 3) Maestras y maestros como agentes de la transformación educativa; 4) Entornos educativos dignos y sana convivencia; 5) Deporte para todas y todos; 6) Rectoría del estado en la educación y consenso social". (PSE, 2020: p.10). Estos seis ejes se agrupan bajo el emblema de la construcción de la "Nueva Escuela Mexicana" en la era de la Cuarta Transformación.

Las acciones y metas de educación universitaria se concentran en la definición de dos problemas básicos: $a$ ) la inequidad en el acceso de los grupos más vulnerables (comunidades indígenas, discapacitados, poblaciones urbanas y rural más pobres), y b) la baja contribución de la educación superior a la movilidad social de los egresados. De esta problematización, se desprenden un conjunto de objetivos y estrategias relacionados principalmente con las prioridades 1, 2 y 6 del PSE, entre los cuales destacan: "promover la revisión de los mecanismos de selección de las universidades"; "ofrecer esquemas flexibles de apoyo para las poblaciones marginadas y más vulnerables"; "crear universidades públicas de vocación comunitaria”; "incentivar a las IES de todos los subsistemas a reforzar el compromiso social con sus comunidades"; "robustecer los mecanismos de evaluación, acreditación y certificación de los programas de educación superior como medida de aseguramiento de la excelencia educativa" (PSE, 2020).

El lenguaje del PSE comparte mucho de la narrativa del viejo régimen de políticas. La narrativa aparentemente rupturista del nuevo gobierno es, en realidad, la continuación de las líneas de acción iniciadas con la modernización y la evaluación de la calidad de las administraciones pasadas. Esta tensión entre el relato transformacionista de la Cuarta Transformación y las prácticas de políticas que se desprenden del programa sectorial sexenal en lo relativo a la educación superior, constituye una dimensión que permite apreciar las diferencias retóricas y las semejanzas prácticas entre el "viejo" y el "nuevo" régimen de políticas universitarias.

\section{Normatividad}

El 15 de mayo de 2019 los congresistas mexicanos aprobaron la iniciativa presidencial de reforma del artículo tercero constitucional que determina las disposiciones generales en el campo de la educación. Esa reforma culminaba con un compromiso de campaña de AMLO: derogar la reforma educativa que el gobierno anterior de Enrique Peña Nieto (2012-2018) había diseñado e instrumentado parcialmente, en medio de conflictos magisteriales y resistencias políticas durante su sexenio.

La reforma desaparecía la evaluación magisterial "punitiva", y junto a ello, al Instituto Nacional de Evaluación de la Educación (INEE), y su sustitución por un "organismo de mejora continua de la educación”. En términos de educación superior, la reforma incluyó la obligatoriedad y gratuidad de toda la educación pública por parte del Estado, que contempla por vez primera, de manera explícita, a la educación terciaria. También consideró la promulgación de una nueva normatividad para ese sector, y en uno de los artículos transitorios, la creación de un "Fondo nacional para el desarrollo de la educación superior" para asegurar la sustentabilidad y certeza presupuestal de las instituciones públicas universitarias. 
Esta es una novedad importante del gobierno lopezobradorista: el impulso a una reforma a la normatividad vigente en la educación superior. Desde los primeros meses de su gestión, comenzó la elaboración de un "Anteproyecto de Ley General de Educación Superior" (2019), en el cual un grupo multidisciplinario de académicos universitarios, representantes de la ANUIES y funcionarios del sector trabajaron, en un proyecto de ley que incluyera las transformaciones y adecuaciones jurídicas necesarias para favorecer un desarrollo equitativo, financieramente sostenible e institucionalmente factible de la educación superior universitaria y no universitaria del país (Rodríguez, 2019).

Aunque la idea de una nueva legislación no es nueva (la propia ANUIES había estado insistiendo en ese tema desde por lo menos en el año del 2012), la búsqueda de una mayor certeza jurídica, financiera y política del desarrollo de la educación terciaria, basada en el respeto a la autonomía universitaria para ese sector específico de la educación superior, fue considerada favorablemente por el gobierno de AMLO. El diagnóstico del proyecto considera que la normatividad vigente -que data de 1978, con la Ley para la Coordinación de la Educación Superior (LCES)se encontraba claramente rebasada por el tamaño, diversidad y complejidad contemporánea del sistema nacional de educación superior.

El proceso de análisis y discusión pública de la propuesta comenzó en junio de 2019 en distintos foros regionales en todo el país. Sin embargo, hasta julio de 2020 no había sido presentada una propuesta formal y definitiva del proyecto a la Cámara de Diputados. La irrupción de la crisis sanitaria y económica provocada por la pandemia de la COVID-19 cambió las prioridades del ejecutivo y el legislativo federal, e interrumpió el proceso de aprobación e instrumentación de la LGES. En esas circunstancias no se vislumbra que en el corto plazo el proyecto de una nueva normatividad pueda ser considerado por los poderes públicos.

\section{Financiamiento}

En términos de las políticas de financiamiento universitario, el nuevo gobierno tampoco se diferencia demasiado de sus antecesores. Un patrón histórico de comportamientos inestables y erráticos en el financiamiento federal se combina con una clara tendencia 
hacia la disminución relativa de los presupuestos anuales destinados a la educación superior. Asimismo, el patrón de negociación presupuestal entre las universidades y el ejecutivo federal confirma una dinámica de ajustes y reasignaciones al proyecto original del ejecutivo, que luego suele ser modificado por la intervención de la Cámara de Diputados.

En el proyecto presupuestario 2019 presentado por el ejecutivo federal se contemplaba inicialmente una reducción real de $6.2 \%$ en los recursos destinados a las universidades federales y de $3.8 \%$ a las universidades estatales. Los fondos extraordinarios eran reducidos 53\% respecto al año anterior, y se reasignaban fondos para los dos programas del nuevo gobierno: Las universidades para el Bienestar (mil millones) y Jóvenes Construyendo el Futuro (4,300 millones) (Mendoza Rojas, 2019a). Luego de la negociación parlamentaria, esas reducciones fueron revisadas, y los recursos se mantuvieron en términos reales al nivel de 2018, es decir, crecimiento cero.

Visto en perspectiva, el financiamiento federal a la educación superior en el primer año de gobierno de AMLO fue equivalente al de 2013 (el primer año de gobierno de Peña Nieto). Si se observa como porcentaje del PIB, en 2019 se destinó $0.54 \%$ en educación superior y posgrado, y $0.77 \%$ si se agregan los recursos para la Ciencia y la Tecnología. Una década antes, en 2009 , se destinaban respectivamente $0.67 \%$ y $0.89 \%$ a esos rubros (Ibid.).

Si se mira desde otros indicadores como el gasto por alumno, la tendencia a la disminución también es clara. La máxima de hacer más con menos (un principio explícito de las políticas económicas neoliberales) se ha "naturalizado" como práctica de las políticas de financiamiento público; más cobertura con menos presupuesto es una constante del sistema mexicano de educación superior, una clara herencia del viejo régimen de políticas. Entre 1988 y 2019 el gasto federal se incrementó en términos reales $164 \%$, mientas que la matrícula de educación superior lo hizo 187\%. Según cálculos de Mendoza Rojas, durante el primer año de gobierno de AMLO (2019) ese gasto equivale a 49,669 pesos por alumno, el menor de todos los primeros años de gobierno de cinco sexenios anteriores: Salinas en 1989 (53,987), Zedillo en 1994 (69,173), Fox en 2001 (65,269), Calderón en 2007 $(63,058)$, y Peña Nieto en $2013(66,619)^{5}$.

\section{La dimensión internacional}

¿Existe articulación de la agenda gubernamental con una agenda regional o internacional? No hay hasta el momento ningún elemento para afirmar que la agenda del lopezobradorismo tenga relación con las agendas internacionales o regionales que se han impulsado desde diversos organismos o instituciones. Ni en el PND ni en el PSE se contemplan acciones o metas específicas al respecto. Las ideas, acciones y retórica de la política educativa universitaria del oficialismo se alejan de los temas impulsados por organismos como la OECD o la UNESCO, o son relativamente indiferentes a los que se discuten en América Latina como el MERCOSUR o UNASUR. Ello obedece al retorno de una suerte de neonacionalismo gubernamental que desconfía de la calidad, la globalización o la internacionalización de la educación superior, de las ilusiones de las "universidades de clase mundial", o de la 
cooperación regional como mecanismo de mejora de la igualdad, justicia o equidad en el acceso a la educación superior.

Son notables estas ausencias en términos de la política pública nacional que se perfila ahora y en los próximos años en México. No obstante buena parte de las universidades públicas federales y estatales mantienen intercambios y comparten agendas con otras universidades, regiones y organismos internacionales, individualmente o a través de organizaciones como ANUIES o la UDUAL. Esa tensión entre los intereses "nacionalistas" de la política gubernamental sobre la educación superior y los intereses "internacionalistas" que mantienen las universidades públicas forma parte de la compleja configuración de un régimen de políticas que mantiene más continuidades prácticas que distancias teóricas o ideológicas con los ciclos de políticas de los gobiernos anteriores.

\section{Consideraciones finales}

Un balance preliminar de la acción del nuevo oficialismo en el campo de la educación superior universitaria indica que no hay evidencias de la construcción de un nuevo régimen de políticas universitarias. Las ideas, arreglos y actores protagónicos conservan fuertes líneas de continuidad con el viejo régimen. La obligatoriedad y la gratuidad coexisten con las ideas de evaluación de la calidad del desempeño universitario y las métricas correspondientes. Los arreglos suponen nuevas ofertas institucionales universitarias (las Universidades para el Bienestar), como ocurrió también en el pasado reciente (Universidades Interculturales, Universidades estatales con apoyo solidario, Universidades tecnológicas). Las políticas de financiamiento público federal puestas en marcha por el gobierno de AMLO heredan del viejo régimen la distinción entre el financiamiento ordinario y el extraordinario, y la competencia entre las universidades públicas por bolsas presupuestarias anuales como incentivos del comportamiento institucional, cuyas reglas de acceso y metas de cumplimiento están fijadas por el Ejecutivo Federal. Finalmente, los actores centrales del régimen son hoy como ayer el Ejecutivo Federal, la Cámara de Diputados y las autoridades de las instituciones públicas federales, desplazando relativamente a actores como la organización representante de las universidades públicas (ANUIES), los gobernadores de los estados, y los organismos acreditadores de la calidad (COPAES).

Las políticas de educación superior son más gubernamentales que públicas, como ocurrió también en el viejo régimen. El diseño e instrumentación de los programas prioritarios, las políticas de financiamiento y la negociación de las políticas tienen una marcada lógica de arriba-abajo, en las cuales el poder ejecutivo tiene un papel central por encima de otros actores interesados. La acción más destacada del nuevo gobierno es el impulso a la reforma del Marco Normativo (la Ley General de Educación Superior), que constituye una novedad importante en el campo de las políticas universitarias. Sin embargo, una externalidad inesperada tanto en su origen como en su magnitud (la crisis sanitaria y económica de la COVID-19), ha causado alta incertidumbre sobre la prioridad y factibilidad de dicha iniciativa tanto para el ejecutivo como para el legislativo. En tales circunstancias, 
todo indica que las prácticas conservadoras del viejo régimen parecen imponerse a la narrativa rupturista de las políticas universitarias del sexenio lopezobradorista. Como ha ocurrido en otros casos nacionales y en diversos campos de la acción pública, las prácticas políticas y de políticas dominan las creencias e ideas del nuevo gobierno nacional.

\section{Notas}

1. La Cuarta Transformación parte de la idea de que la historia mexicana ha experimentado tres grandes cambios: la Independencia (1810-1821), la Reforma (1855-1863), y la Revolución (1910-1917), y el nuevo gobierno se presenta como la "Cuarta Transformación Nacional" (2018-2024). En palabras de AMLO al asumir la Presidencia de la República el 1 de diciembre de 2018: "Si definimos en pocas palabras las tres grandes transformaciones de nuestra historia, podríamos resumir que en la Independencia se luchó por abolir la esclavitud y alcanzar la soberanía nacional, en la Reforma por el predominio del poder civil y por la Restauración de la República, y en la Revolución nuestro pueblo y sus extraordinarios dirigentes lucharon por la justicia y la democracia. Ahora, nosotros queremos convertir la honestidad y la fraternidad en forma de vida y de gobierno". http://www.educacionfutura.org/la-cuarta-transformacion

2. Habría que agregar entre las nuevas instituciones universitarias del gobierno de AMLO la creación de la "Universidad de la Seguridad Pública" y la "Universidad de la Salud". La primera fue inaugurada en julio de 2019 como parte de la estrategia de seguridad que dio origen a la Guardia Nacional. La nueva institución es federal, forma parte del Consejo Nacional de la Seguridad Pública, y tiene como objetivo la formación y capacitación de los miembros de la Guardia Nacional (Acosta, 2019b). La segunda inició sus operaciones en julio de 2020, y depende del Gobierno de la Ciudad de México, con el apoyo de la Secretaría de Salud del gobierno de AMLO.

3. Varios estudios recientes muestran evidencias positivas de las universidades públicas en indicadores como equidad en el acceso, inserción laboral y movilidad social. Señalan también déficits importantes en estos indicadores entre diversas carreras, disciplinas y universidades. Sin embargo, en los documentos oficiales del nuevo gobierno, no se hace mención alguna a esos estudios y datos.

4. Esta meta significa que la matrícula total del sistema de educación debería crecer anualmente al doble de lo experimentado en el periodo 2000-2018. En los tres sexenios anteriores, la matrícula incorporó un promedio de 145 mil estudiantes por año. Ello se acompañó en el mismo periodo de la contratación de 8.862 nuevos puestos académicos por año y la apertura de 125 establecimientos de educación superior anualmente, tanto públicos como privados.

5. A pesos constantes de 2019 . 


\section{Referencias}

Acosta Silva, Adrián (2020). “Autonomía universitaria y estatalidad”, Revista de la Educación Superior, 49(193) enero-marzo 2020, ANUIES, México: Pp. 1-23. http://resu.anuies.mx/ojs/index. $\mathrm{php} / \mathrm{resu} / \mathrm{article} / \mathrm{view} / 1025 / 415$

Acosta Silva, Adrián (2019a). “La épica de los indicadores”, en Lomelí Vanegas, Leonardo y Roberto Escalante Semerena (Coordinadores), Autonomías bajo acecho, Siglo XXI Editores, México: Pp. 83-97.

Acosta Silva, Adrián (2019b). "Un asunto de Estado: la Universidad de la Seguridad Pública”, Campus-Milenio, 18 de julio, 2019.

http://campusmilenio.mx/index.php?option=com_k2\&view=item\&id=17280:la-universidad-de-la-seguridad-publica-un-asunto-de-estado\&Itemid=349

Acosta Silva, Adrián (2014). "Gobierno universitario y comportamiento institucional: la experiencia mexicana, 1990-2012”, Bordón, Vol. 66, Núm.1, enero-marzo, Madrid: Pp. 31-44. (consultado el 10/06/2020)

https://recyt.fecyt.es/index.php/BORDON/article/view/Bordon.2014.66102

Acosta Silva, Adrián (2010). Príncipes, burócratas y gerentes. El gobierno de las universidades públicas en México, ANUIES/UdUAL, México, $2^{\mathrm{a}}$. Ed.

Acosta Silva, Adrián, y Jordi Planas Coll, (Coords.) (2014). La arquitectura del poliedro. Itinerarios universitarios, equidad y movilidad social en México, CUCEA-Universidad de Guadalajara.

Anteproyecto de Ley General de Educación Superior (Octubre, 2019). https://consulta-ley-educacion-superior.mx/wp-content/uploads/2019/11/ANTEPROYECTO-LGES-Octubre-2019.pdf

ANUIES (2018). Visión y acción 2030. Propuesta de la ANUIES para mejorar la educación superior en México, México.

https://visionyaccion2030.anuies.mx/Vision_accion2030.pdf

Capano, Giliberto (2011). "Government continues to do its job: a comparative study of governance shifts in the higher education sector”, Public Administration, 89 (4), Pp. 1622-1642.

López Obrador, Andrés Manuel (2017). 2018 La salida. Decadencia y renacimiento de México, Planeta, México.

May, Peter J. (2018). "Reconsiderando los fracasos en la implementación: la perspectiva de los regímenes de políticas públicas”, en Pardo, María del Carmen, Mauricio.I. Dussauge L. y Guillermo M. Cejudo (Eds.), Implementación de políticas públicas. Una antología, CIDE, Pp. 283-318.

Mendoza Rojas, Javier. (2019a). "Presupuesto federal de educación superior en el primer año del gobierno de Andrés Manuel López Obrador: Negociaciones y retos”. Revista de la Educación Superior, 48(191): Pp. 51-82.

Mendoza Rojas, Javier (2019b). "Proyecto Presupuesto Federal 2019 para educación superior", Seminario de Educación Superior, UNAM.

https://www.ses.unam.mx/integrantes/uploadfile/jmendoza/Mendoza2019_PresentacionSES.pdf

Poder Ejecutivo Federal (2019). Plan Nacional de Desarrollo 2019-2024, México. https://www.planeacion.sep.gob.mx/Doc/PND.pdf (consultado el 09/06/2020)

Rodríguez, Roberto (2019). “Hacia la Ley General de Educación Superior”. Educación Futura. http:// www.educacionfutura.org/hacia-la-ley-general-de-educacion-superior/

Secretaría de Educación Pública (2020). Programa Sectorial de Educación 2020-2024, México. https://www.gob.mx/cms/uploads/attachment/file/562379/Decreto_por_el_que_se_publica_el_PSE_2020-2024.pdf

Secretaría de Educación Pública, SES/Dirección General de Educación Superior Universitaria (DGSU) (2020). "Programas de Educación Superior", México. (Consultado el 15/06/2020). https://www.dgesu.ses.sep.gob.mx/Indice.html\#Span1

------Subsecretaría de Educación Superior (2019), sitio oficial https://www.educacionsuperior.sep.gob.mx

Wilson, C. (2000). "Policy Regimes and Policy Change”, Journal of Public Policy, 20, Pp. 247-274, Cambridge. 